\title{
Wideband signals for Phase Differencing Sonar Systems
}

\author{
Jitendra Singh Sewada \\ Univ. Grenoble Alpes, CNRS, \\ Grenoble INP*, GIPSA-Lab, \\ 38000 Grenoble, France \\ *Institute of Engineering Univ. \\ Grenoble ALpes \\ jitendra-singh.sewada@gipsa- \\ lab.grenoble-inp.fr
}

\author{
Cornel Ioana \\ Univ. Grenoble Alpes, CNRS, \\ Grenoble INP*, GIPSA-Lab, \\ 38000 Grenoble, France \\ *Institute of Engineering Univ. \\ Grenoble ALpes \\ Cornel.Ioana@gipsa- \\ lab.grenoble-inp.fr
}

\author{
Matt Geen \\ ITER-Systems \\ 310 Impasse de la Tuilerie, \\ 74410 Saint-Jorioz, France \\ matt.geen@iter-systems.com \\ Jérôme Mars \\ Univ. Grenoble Alpes, CNRS, \\ Grenoble INP*, GIPSA-Lab, \\ 38000 Grenoble, France \\ *Institute of Engineering Univ. \\ Grenoble ALpes \\ jerome.mars@gipsa- \\ lab.grenoble-inp.fr
}

\begin{abstract}
This research work deals with the resolution problem in Phase Differencing Bathymetric Sonar Systems (PDBS), also called interferometric sonars ("Interferometers"). Wideband signals are compared with the narrowband continuous wave $(\mathrm{CW})$ pulses to assess the relative merits of signals to improve the range and angle resolution. The idea is inspired by the marine mammals, who use complex wideband signals for navigation and target detection. A set of waveforms e.g. CW Pulses, LFM (Linear Frequency Modulated) Pulses, EFM (Exponential Frequency Modulation) Pulses were used with Bathyswath-1 transducers for the experiment. A comparative study was done to assess the set of signals for range and angular resolution of Interferometers. Wideband signal processing techniques are used to solve the problem of trade-off between range and resolution. This paper gives an introduction to interferometry sonars, the major problems with them and the wideband processing approach to improving them.
\end{abstract}

Keywords-Active Sonar, Interferometric Sonar, Phase Differential Bathymetric Sonar, Bathymetry, Wideband signals, Matched Filtering

\section{INTRODUCTION}

High resolution images of Earth's surface are easily provided by radar and satellite photographs, but the view underwater is still blurry. Yet there is a need for high resolution seabed maps and 3D images for underwater studies for both research and industrial purposes. In recent years, there have been significant developments in the underwater surveying equipment. Interferometric swath sonar is one of them and is widely used for bathymetry surveys. Swath bathymetry sonar systems are the preferred tool in shallow waters, due to the longer swath range compared to typical multibeam echosounders. A Multi beam echosounders (MBES) is limited by the fixed swath angle under the boat and has smaller range in shallow water. A MBES uses beamforming technique to create a set of beams and detects the range in each beam at a fixed observation angle [4]. On other hand, interferometry systems are not limited by the fixed angle problem so they can calculate the depth values for any received signal form any possible direction. But still the interferometers are not preferred system due to higher uncertainty in angle calculation. Here we tried to study and find possible solutions to those problems and to improve Interferometric systems. The first thing to do in this study is to find the problems and limitations of Interferometric system, then a comparative study is done with the narrow band (conventional) processing techniques and wideband processing techniques to improve the Interferometer's overall performance.

We used Bathyswath-1 single sonar transducer for the experiments, provided by the ITER Systems. The resonant frequency of the transducer is $468 \mathrm{kHz}$. The experiment took place in a water tank but real world data was also used to identify the problems with existing interferometric technology.

A basic introduction is given in section II to understand the mechanism of Interferometric systems and their history. Section III covers the theoretical background to the problems and solutions. Section V covers the details of our experiments and their results and these results are concluded in section VI.

\section{INTERFEROMTERY AND HISTORY}

\section{A. Interferometry}

The name "Interferometer" comes from the initial use of the interference patterns from sidescan sonar to predict the angle of arrival [2]. The first system to use phase differences to calculate the angle of arrival was called "bathymetric sidescan sonar" by Denbigh in 1977 [1]. Interferometric sonar systems are also known Phase Differencing Bathymetric Sonar (PDBS) system. They measure the phase difference of the signals on two or more closely spaced receivers.

Figure 1 shows the basic setup of interferometry sonar to calculate range and angle from backscattered data. Time since transmit and sound velocity are used to compute the range to the seabed and the phase content of wavefront is used for angle.

Interferometric Equation;

$$
\theta=\theta_{s} \mp \sin ^{-1}\left(\frac{\lambda \Delta \varphi}{2 \pi B}\right)
$$

Where $\theta_{\mathrm{s}}$ is tilt angle of the transducer; $\Delta \varphi$ is phase difference at two receive staves; and $\mathrm{B}$ is the vertical distance between two receive staves.

The first known Bathymetric Sidescan Sonar (BASS) used two receive arrays with $410 \mathrm{kHz}$ operating frequency, placed at three wavelengths apart and used one separate transmit array [1]. 
The signal intensity at any of the receivers was used to create a sidescan image. Later, a system described by Cloet et al. in 1982,

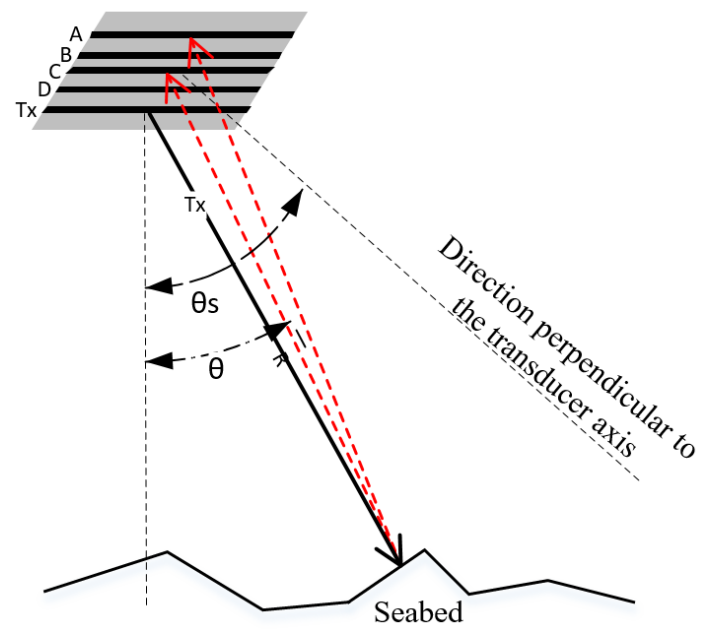

Figure 1 Basic setup of Interferometric Sonar System

operated at $303 \mathrm{kHz}$ and used two widely spaced pairs of receiver arrays and gave a considerable improvement in accuracy [6]. This was called the Vernier technique, it is still used in most Interferometric systems, but with smaller array separation and increased number of pairs.

We are using an Interferometric system called Bathyswath, provided by ITER Systems for our studies. The resonant frequency of the transducer is $468 \mathrm{kHz}$ and there are 4 receive staves and one transmit stave with $1.1^{\circ}$ azimuth beam width. To get around with the problem of angle ambiguity and angle uncertainty, the Vernier method is used in Bathyswath systems. It has only four receive staves with different spacing but more artificial spacing can be generated by subtracting the phase differences from one stave pair from another.

Figure 2 shows a cross-profile view of the spread of depths and sonar noise. Blue data is filtered showing the location of seabed and the red points are rejected points. The depth under transducer is 3.5 meters but the slant range is up to 30 meters each side. This gives an idea that why long range with good resolution is important. Interferometric systems were designed to save the survey cost in shallow water systems where multibeam echosounders achieve a smaller swath range.

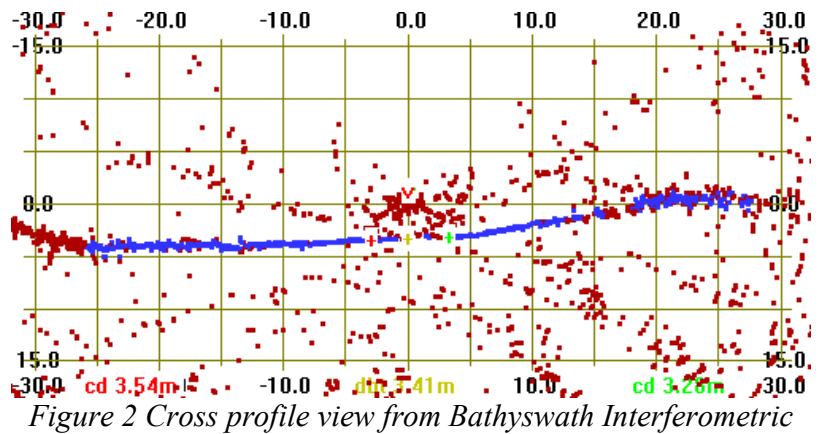

Sonar System

\section{PROBLEMS OF RANGE AND ANGULAR RESOLUTION}

\section{A. Range Resolution}

Across track (range) resolution depends on the sonar frequency used. Range resolution increases with frequency, but higher frequencies give smaller range due to higher absorption loss in water. For fixed frequency sonar systems, we can achieve adequate range by increasing the pulse length of the transmitted signal, but long pulse lengths compromise the range resolution of system.

Range resolution of a $\mathrm{CW}$ system for a pulse length in seconds is given by;

$$
\Delta R=\frac{c \times \text { Pulse Length }}{2}
$$

\section{B. Range ambiguity}

If there are two reflected sound waves from two different targets arrives at same time can cause range ambiguity. Thus, Interferometers are not known to be best tool while looking at the complex structures. Current system uses more than two staves to detect this situation and then the data can be ignored or removed.

\section{BACKGROUND THEORY}

\section{A. Continous Wave Signals}

$\mathrm{CW}$ (Continuous Wave) pulses are widely used in the Interferometers because it gives low complexity in the system design. CW signals are limited by the trade-off between pulse length and range resolution.

To cover a long range with the system, we have to transmit a longer pulse, to put more energy into the water. The Bathyswath sonar system uses rectangular pulses. More acoustic energy increases the signal-to-noise ratio (SNR), which improves angular accuracy. Therefore the sonar range and angular accuracy are inversely related to range resolution. .

\section{B. Wideband Singnals}

\section{1) $L F M$}

Linear frequency modulated pulse waveform are a popular choice in radar systems. Also known as chirp signals, these are widely used in pulse compression techniques. Using wideband pulses breaks the inverse relationship between range resolution and target detection capabilities.

$$
f(t)=f_{0}+k t
$$

Where $\mathrm{k}$ is chirp rate given by $\frac{f_{1}-f_{0}}{T}, f_{1}$ is final frequency $f_{0}$ is initial frequency, and $T$ is the chirp length in seconds.

Time domain function of linear sinusoidal chirp:

$$
x(t)=\sin \left(\phi_{0}+2 \pi\left(f_{0} t+\frac{k}{2} t^{2}\right)\right)
$$

Where $\phi_{0}$ is initial phase 


\section{2) $E F M$}

In Exponential Frequency Modulated (EFM) or Exponential chirps, the frequency component of the signal varies exponentially as a function of time.

$$
f(t)=f_{0} k^{t}
$$

Where $\mathrm{k}$ is chirp rate given by $\left(\frac{f_{1}}{f_{0}}\right)^{\frac{1}{T}}$

Corresponding time domain function of exponential sinusoidal chirp

$$
x(t)=\sin \left(\phi_{0}+2 \pi f_{0}\left(\frac{k^{t}-1}{\ln (k)}\right)\right)
$$

\section{EXPERIMENT}

\section{A. Setup}

A small tank size of one cubic meter is used for the experiments. A ladder shaped target is placed in-front of the transducer at the distance of $60 \mathrm{~cm}$. Target is made of adjustable cylindrical rungs of $28 \mathrm{~mm}$ diameter with the minimum separation of $1 \mathrm{~cm}$.

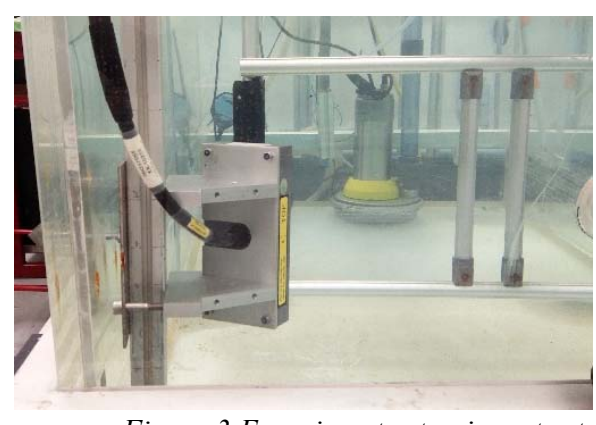

Figure 3 Experiment setup in water tank and target

\section{B. Processing Techniques}

\section{1) Matched Filtring}

Matched filtering (MF) is widely used for signal detection in radar and sonar. MF maximizes the signal to noise ratio (SNR) of the signal being detected with respect to noise. Output of MF is obtained by correlating a known signal with the unknown signal. In our case the known signal is the transmitted reference signal and the unknown signal is the target reflected signals.

A longer pulse has more energy so the output of MF will give a higher SNR. But with a longer pulse the side lobes start to dominate in output. [9].

Output of matched filter is given by

$$
y(t)=\int_{T}^{0} r(t) h(T-t) d t
$$

The above equation is cross-correlation between received signal $r(t)$ and reference signal $h(t)$. This results in the maximised signal to noise ratio.

\section{Observation}

1) $\mathrm{CW}$

The current Bathyswath is a single frequency, or Continuous Wave $(\mathrm{CW})$ system. It transmits at a fixed frequency and receives with a bandwidth of up to $100 \mathrm{kHz}$.

From the Figure 4 and Figure 5, we can clearly establish an inverse relationship between pulse length and range resolution. With a 2-cycle $(0.006 \mathrm{~m})$ long pulse we can easily see both rungs of the ladder but the received signal energy is low. If we increase the pulse length to 10 or 20 cycles then we increase the received signal energy but we start to lose the spatial separation between the rungs.
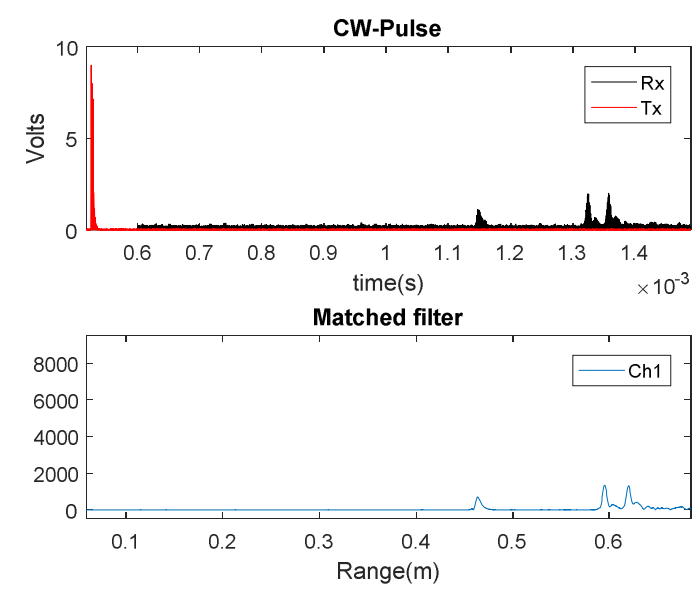

Figure 4 Matched filter output for 2 cycles long $C W$ pulse
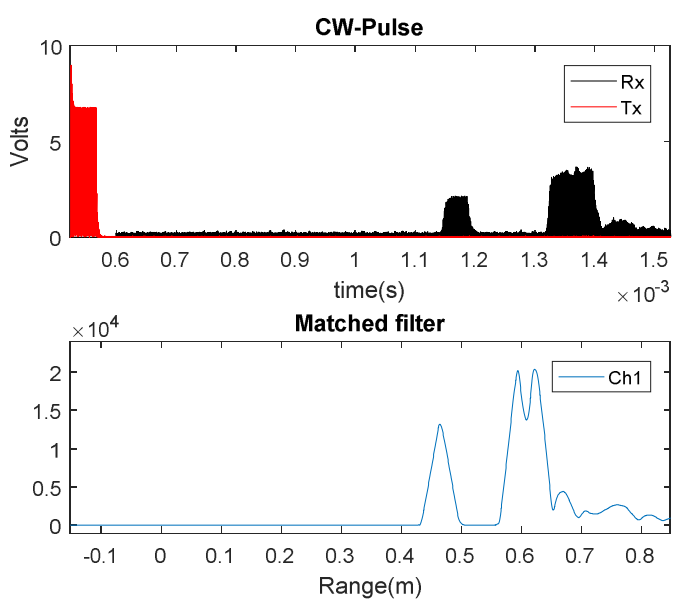

Figure 5 Matched filter output for 20 cycles long CW pulse

Higher frequency systems are preferable for generating high resolution sidescan images but due to the high trade-off between range and resolution, we need to think of alternatives. Fig. 6 shows a sidescan image taken with the $468 \mathrm{kHz} \mathrm{CW}$ system. To cover a range of $60-80$ meters, a $0.12 \mathrm{~m}$ ( 40 cycles) long pulse was transmitted. 


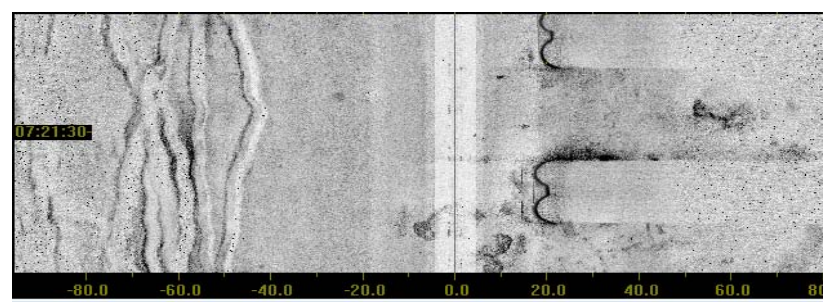

Figure 6 Sidescan image taken with $468 \mathrm{kHz} \mathrm{CW}$ sonar system

\section{2) $L F M$}

Figure 7 and Figure 8 show the results for linear chirp signals with different pulse lengths. Experiment setup and equipment are same as the $\mathrm{CW}$ experiments.
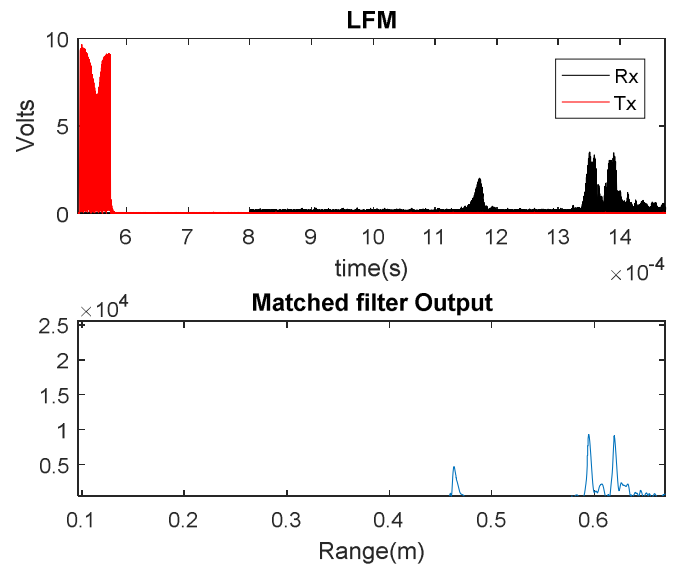

Figure 7 MF output of LFM signal

The matched filter output increases with the increase in bandwidth, and the width of output peaks becomes narrower, which gives increased resolution. The range resolution for a linear chirp is given by following equation:

$$
\Delta R=\frac{\text { Speed of Sound }}{2 \times \text { Bandwidth }}
$$
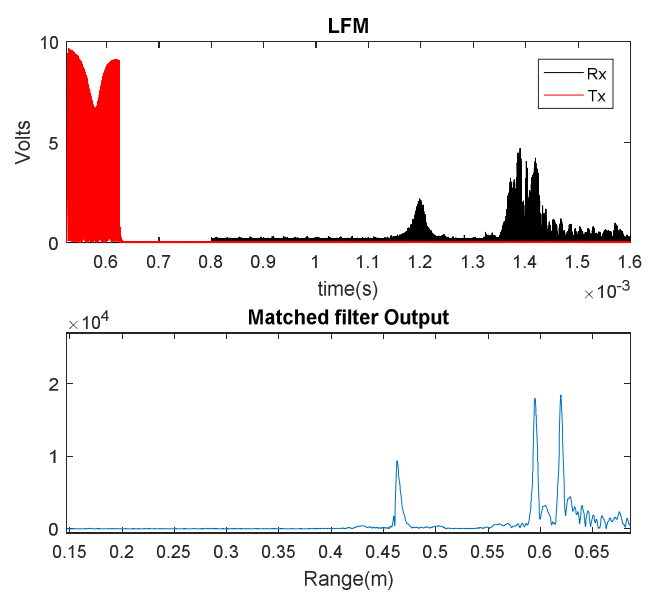

Figure 8 MF output of longer LFM signal
In the underwater environment the signal strength at receiver decreases with the increase in range, due to spreading losses and absorption in the water. We need to increase the chirp length to have a uniform signal strength over different depths and ranges. But in shallow water, increase in pulse length doesn't improve the SNR, because the side lobes start to dominate the correlation noise [9].

In Figure 8, we can see that the signal is overlapped at the front end of receiver but the output of the matched filter is still able to distinguish the both target rungs in the matched filter output.

\section{3) $E F M$}

Exponential chirps have no improved results over linear chirps. Use of EFM pulses adds complexity to the system design, so linear chirps (LFM) are preferable for the implementation in the system.
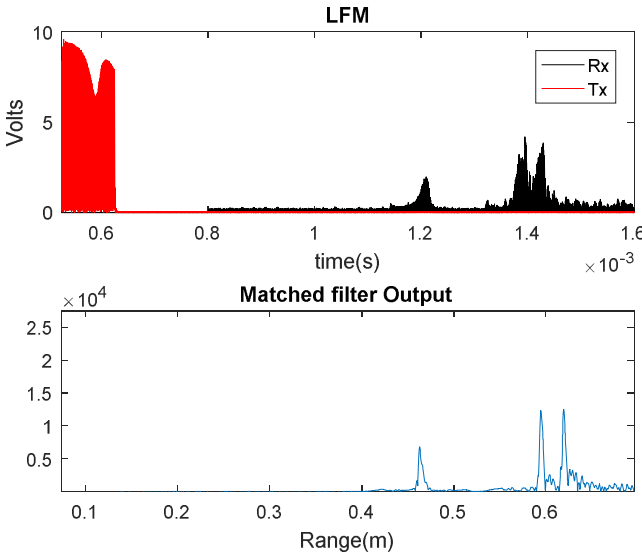

Figure 9 MF output for EFM signal

\section{CONCLUSION}

We used different pulse length for $\mathrm{CW}$ pulses to explain the trade-off between range and resolution. The shortest pulse used was $0.006 \mathrm{~m}$ ( 2 cycles) and was able to detect the both the rungs of target but the SNR value was low. Then we used $0.03 \mathrm{~m}$ (10 cycles) and $0.06 \mathrm{~m}$ (20 cycles) transmitted pulses, which gave resolution of the rungs.

From the experiments, we show that the resolution of a sonar system can be improved significantly with the use of wideband signals. With the $\mathrm{CW}$ pulses the range resolution directly depends on the pulse length: the smaller the pulse higher the resolution but small duration pulses contain less energy, and so range and angle measurement accuracy are limited. With wideband signals, the resolution is unaffected by pulse length. Long wideband pulses give benefits of long range. We also conclude that the results of EFM and LFM pulses are almost same in this case, which gives benefits to LFM signal because of lower complexity in hardware design. Classical Matched Filtering technique is used to detect the signal component from a noisy received signal. The output of a signal generator was used as the reference signal, but in the real world application it is more complicated to get the reference acoustic signal, so 
different techniques need to be considered to recover the transmitted reference signal.

Further developments will consider adaptive pulse and wideband interferometry experiments.

\section{REFERENCES}

[1] P.N. Denbigh, "A bathymetric sidescan sonar", in proc. Ultrasonics Int. 79 Conf., 1979

[2] M.J.P. Heaton and W.G. Haslett, "Interpretation of Lloyd mirror in sidescan sonar", Proc. SOc. Underwater Tech., vol. 1, no. 1, pp. 24-38, 1971.

[3] Philip N Denbigh, "Swath Bathymetry: Principles of Operation and an Analysis of Errors", in IEEE Journal of Oceanic Engineering, Vol.14 1989
[4] X. Lurton, "Theoretical Modelling of Acoustical Measurment Accuracy for Swath Bathymetric Sonars", International Hydrographic Review, vol. 4 No. 2,2003

[5] X. Lurton, "Swath Bathymetry using phase differences: theoretical analysis of acoustical measurment percisiion", IEEE Journal of Oceanic Engineering, vol. 1, pp. 351-363, 2000

[6] R.L. Cloet, S.L. Hurst, C.R. Edwards, P.S. Phillips, and A.J. Duncan, "A sideways looking towed depth measuring system”, J. Roy. Inst. Nav., vol. 35, pp. 411-420, Sept. 1982

[7] P.N. Denbigh, "Phase only side-scan sonar for underwater mapping ", Acoustic letters, vol. 1, pp. 84-87, 1977.

[8] P. H. Kraeutner and John S. Bird, "Beyond interferometry, resolving multiple angles-of-arrival in Swath Bathymetric Imaging ", Oceans'99, vol. 1, pp. 37-45, 1999

[9] Md. J. Alam, E. H. Huntington, M. R. Frater, "Relationship between length of the chirp and depth of the ocean in active sonar processing", IEEE 2013 